\title{
LEVER-PULLING RESPONSE SPEED IN GHILDREN AS A FUNCTION OF DELAY OF REINFORCEMENT AND INGENTIVE VALUE
}

\author{
YOSHIO SHIROIWA ${ }^{1}$ \\ Kinjo Gakuin University
}

\begin{abstract}
The speed of a lever-pulling response was measured for 48 kindergarten children under different conditions of delay of reinforcement and incentive value. Ss were divided into 6 groups depending upon 3 delayed reinforcements (Immediate, 5 -sec and 10-sec Delay) and 2 incentive values (High and Low). On each trial, measures of starting and movement time of a leverpulling response were taken. The starting speeds were inversely related to the delay of reinforcement. For the movement speeds, Ss of the immediate reinforcement groups responded faster than those of the delayed reinforcement groups. The incentive value variable did not, however, affect both speeds. The data were interpreted in terms of the competing response hypothesis.
\end{abstract}

Spence (1956, p. 116-118) has suggested that, in a straight alley situation with rats, their starting speed differences mainly reflect the effect of " competing responses," while their movement speed differences mainly reflect the effect of motivational variables. A few investigators (Rieber, 1961; Rabinowitz, 1966) have applied Spence's notion to children's lever-pulling situation under different conditions of delay of reinforcement that facilitates the association of extraneous responses which are incompatible with the instrumental response itself. Starting speed, in such a situation, is defined as the reciprocal of $S$ 's response time, while movement speed as the reciprocal of the time taken by $S$ to pull the lever through its full sweep.

1 The author is greatly indebted to Professor T. Naito of Kinjo Gakuin University for his valuable comments and critical reading of the manuscript. The author also would like to express his deep gratitude to $\mathrm{Mr}$. W. P. Baldwin for editing the English phraseology of the manuscript, and to Miss K. Mizuno of Kariya Kindergarten for her helpful assistance in conducting the present experiment. Requests for reprints should be sent to Yoshio Shiroiwa, Kinjo Gakuin University, Omori, Moriyama-ku, Nagoya 463 , Japan.
Generally, the results of these investigators have shown to be consistent with the above Spence's theory.

Shiroiwa (1975) has also examined the effects of delay of reinforcement and incentive value on children's lever-pulling response for the purpose of testing the Spence's theory. The results of the study have shown that the longer the delay interval of reinforcement, the less the starting and the movement speeds, and the higher the incentive value, the greater the movement speed. The purpose of the present study is to attempt to replicate and extend our previous findings by determining whether or not rather shorter delay interval of reinforcement, $5 \mathrm{sec}$, affects the speed of children's lever-pulling response under different incentive value conditions from those of the previous study in which the incentive variable has been controlled by the secondary reinforcement.

\section{MethoD}

\section{Subjects}

The $S$ s used in the present study were 48 experimentally naive kindergarten children who ranged in age from 60 to 78 months (mean 
$\mathrm{CA}=70.3$ months, $S D=5.2$ months).

\section{Apparatus}

The apparatus consisted of a gray plywood box, approximately $42 \times 40 \times 21 \mathrm{~cm}$. The upper half of the front panel stood vertically and the lower half sloped slightly towards $S \mathrm{~s}$. A lever protruded $9 \mathrm{~cm}$ from the right half of the lower part of the front panel. A short plastic tube was fastened to the end of the lever, which provided a gripping surface. The lever could be moved downward a total distance of $8 \mathrm{~cm}$. A red hand pattern located at the bottom righthand corner of the lower front panel provided a common starting point for all $S$ s. On the left half of the lower front panel, there was a $21 \times 7$ $\mathrm{cm}$ visible diagonal alley through which a poker chip was rolled down. A goal box, located at the end of the alley, was covered with transparent plastic so that $S$ could see the accumulation of the poker chips but was prevented from handling them. The signal for $S$ to pull the lever was a red stimulus light (CS). This light was composed of 16 light-emitting diodes (SL-103) which were circularly arranged (3.5 $\mathrm{cm}$ in diameter) in the $7 \times 7 \times 1.5 \mathrm{~cm}$ black box located in the center of the upper part of the front panel.

The initial trial was started by the pressing of a start-button on the programming equipment and afterward, the CS was automatically turned on by the programming equipment in accordance with a predetermined intertrial interval. A complete depression of the lever offset the CS and activated an electric interval timer which controlled the automatic poker chip dispenser device attached inside the apparatus. This allowed automatic control of the delay interval. Two electric timers were wired into the circuit. The first timer was started as soon as the CS was turned on. When $S$ started to pull down the lever from the resting position, the first timer was stopped and the second timer was started. As soon as the lever was pulled down through its $8 \mathrm{~cm}$ excursion, the second timer was stopped, and the CS was turned off. Both timers were automatically reset just before the next trial was started. Time recorded by the first timer was referred to as starting time, and time recorded by the second timer, as movement time. Both times were recorded to the nearest $.01 \mathrm{sec}$.

\section{Procedure}

Fourty-eight $S$ s were randomly divided into six experimental groups with the single restriction under which four males and four females should be assigned to each group. The experimental design was a $2 \times 3$ factorial design according to delay of reinforcement and incentive value conditions. The six groups were Group High-Immediate (H-IM), Group High-5-sec Delay $\left(\mathrm{H}-\mathrm{D}_{5}\right)$, Group High-10-

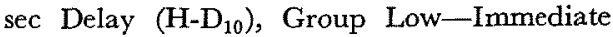

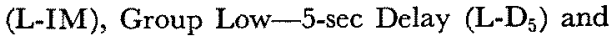
Group Low-10-sec Delay $\left(\mathrm{L}_{-10} \mathrm{D}_{10}\right)$.

The $S$ s in Groups IM were reinforced with a poker chip immediately after their response to the CS. The $S \mathrm{~s}$ in Groups $D_{5}$ and $D_{10}$ were given 5- and 10-sec period of delay between their response to the CS and delivery of the reinforcer, respectively.

The $S$ s were individually led into a utility room used as an experimental room in the kindergarten. Prior to the lever-pulling training, the $S \mathrm{~s}$ assigned to Groups $\mathrm{H}$ were shown a collection of ten toys spread on a table and were asked to choose any one toy which they would prefer to win the "game " to be played. The $S \mathrm{~s}$ in Groups L were merely informed that they could win a string of $30 \mathrm{~cm}$ long $(.7 \mathrm{~cm}$ in diameter) and had no opportunity to state a preference.

Then, each $S$ of the groups was introduced and seated at the lever-pulling apparatus, and was asked to play "poker-chip game." The $E$ explained to $S$ that the chosen toy (Groups $H$ ) or a string (Groups L) could be won if enough chips were acquired in the goal box. The $S$ was instructed that if he pull the lever down once with his right hand when the red stimulus light was turned on, his response would be followed by ejection of a poker chip in the goal box. He was also asked to watch the CS box and to keep his hand on the red hand pattern when the CS was not on. After two reinforced practice trials, the chosen toy or the string was located to the left of the goal 
box and 40 trials were given to all $S \mathrm{~s}$. The interval between successive stimulus presentations (intertrial interval) was varied at random from 18 to $22 \mathrm{sec}$ (mean=20 sec) for all groups including the delay interval in Groups $\mathrm{D}_{5}$ and $\mathrm{D}_{10}$. The $E$ said "start" approximately $3 \mathrm{sec}$ before the initial onset of the CS, and afterward, no ready signal was give to the $S$ throughout the experiment.

No $S$ s were allowed to take their acquired incentives out of the experimental room. After the completion of the present experiment, each $S$ of all groups received a similar inexpensive toy as remuneration for his service to the present experiment.

\section{Results}

All the data were transformed by taking the reciprocal of the starting time and the movement time measures for each $S$ on each trial and multiplying it by 100 , yielding starting speeds and movement speeds. Results of both speeds were shown as a function of 5 blocks of trials: The first 10 trials were divided into two blocks of 5 trials, and the remaining 30 trials into 3 blocks of 10 trials.

\section{Starting Speeds}

Figure 1 presents the mean starting speed for the two incentive-value groups separately as a function of 5 blocks of trials. The unjointed points on the figure represent the mean speeds for the first trial of the six groups. A simple analysis of variance performed on the data for trial one showed that the starting speeds of these groups did not differ significantly prior to differential treatment $(F=1.10$, $d f=5 / 42, p>.05)$. A Type III analysis of variance (Lindquist, 1953) was performed on the starting speeds with delay of reinforcement and incentive value as the between- $S_{\mathrm{s}}$ variables and trial blocks as the within-Ss variable. The main effect of delay of reinforcement was of significance $(F=8.27, d f=2 / 42, p<.01)$. Further analyses on this effect showed that $S$ s in Groups IM responded significantly faster than those in Groups $D_{5}$ and $D_{10}$ $\left(\mathrm{IM}>\mathrm{D}_{5}: \quad F=4.51, \quad d f=1 / 42, \quad p<.05\right.$; $\left.\mathrm{IM}>\mathrm{D}_{10}: \quad F=18.90, d f=1 / 42, \quad p<.01\right)$, and $S_{s}$ in Groups $D_{5}$ responded significantly faster than those in Groups $D_{10}$ $(F=4.31, d f=1 / 42, p<.05)$. The trial blocks effect was also significant $(F=5.52$, $d f=4 / 168, p<.01)$. The main effect of incentive value, however, was not significant. None of the interaction effects was also significant.

\section{Movement Speeds}

Mean movement speeds are shown in

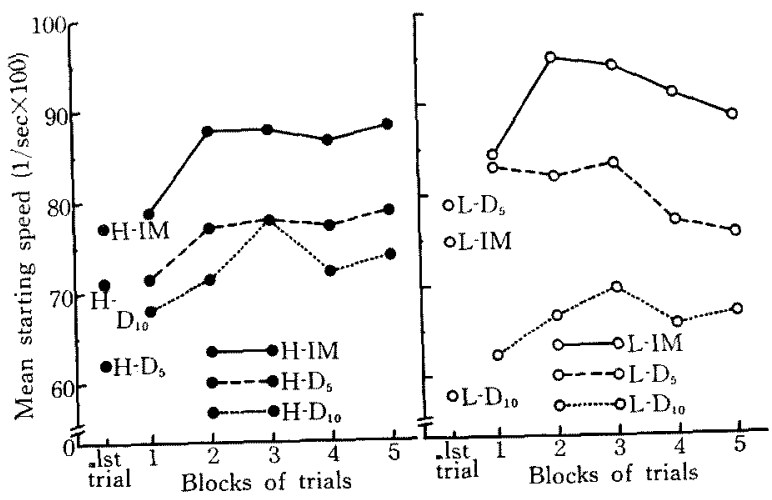

FIG. 1. Mean starting speed for three high-incentive groups (left panel) and for three low-incentive groups (right panel) as a function of blocks of trials. 
Fig. 2 as a function of 5 blocks of trials for the two incentive-value groups separately. The unjointed points on the figure represent the mean speeds for the first trial of the six groups. There was also no significant difference between the mean of the six groups on the first trial with respect to this measure $(F=1.41, d f=5 / 42, p>$ $.05)$. The Lindquist's Type III analysis of variance was similarly conducted on the movement speeds. Significant effect was obtained for delay of reinforcement $(F=$ $7.70, d f=2 / 42, p<.01)$. Further analyses on this effect indicated that $S \mathrm{~s}$ in Groups IM responded significantly faster than those in Groups $D_{5}$ and $D_{10}\left(I M>D_{5}\right.$ : $F=5.56, \quad d f=1 / 42, \quad p<.05 ; \quad$ IM $>\mathrm{D}_{10}$ : $F=15.18, d f=1 / 42, p<.01)$. The difference between Groups $D_{5}$ and $D_{10}$, however, was not significant. Significant effects were not found for incentive value, and for the interaction of delay of reinforcement and incentive value.

As to the effect of trial blocks and the interaction effects of this variable with the other variables, the trial blocks effect, and the interaction of trial blocks and delay of reinforcement were significant $(F=6.18$, $d f=4 / 168, p<.01$ and $F=4.23, d f=8 / 168$, $p<.01$, respectively). These results as a whole indicated that the mean movement speeds of Groups IM increased from the first block to the third block and afterward, maintained the level of performance over the remaining blocks of trials, while Groups $D_{5}$ and $D_{10}$ maintained the same level of performance over all blocks of trials, as seen in Fig. 2.

\section{Discussion}

It appears obvious from the data presented above that the starting speeds are inversely related to the delay of reinforcement. This result may be readily accounted for by Spence's interpretation (1956, p. $116 \mathrm{ff}$.) that the starting speeds are inversely related to the number of competing responses aroused by the CS. That is, the longer the delay interval of reinforcement, the more have the competing responses developed and therefore, the poorer the performance. The fact that the starting speed is less in the delayed reinforcement groups than in the immediate reinforcement groups is consistent with findings of Rieber (1961), Rabinowitz (1966) and our previous study (Shiroiwa, 1975). Moreover, the present result that the incentive conditions have not differentially affected the starting speed is also in line with Rabinowitz's and our previous findings. These facts, taken together, are completely consistent with the

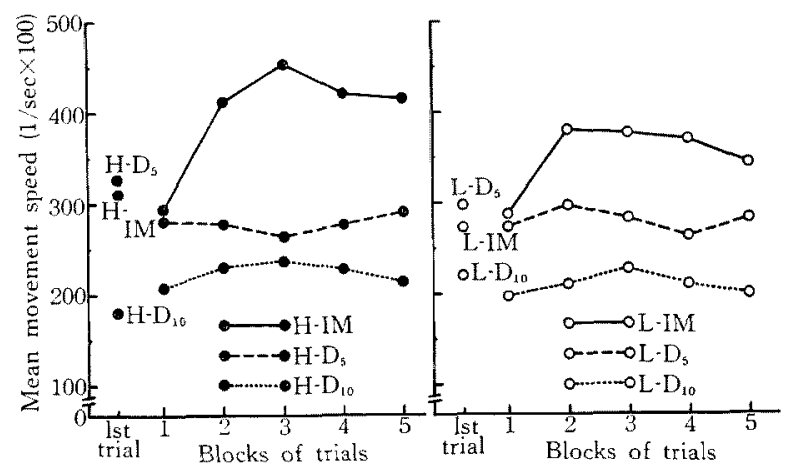

Frg. 2. Mean movement speed for three high-incentive groups (left panel) and for three low-incentive groups (right panel) as a function of blocks of trials. 
Spence's theory (1956) showing that the starting speed mainly reflects the effect of competing responses but not the effect of motivational variables.

The fact that $S$ s in Groups IM have shown a greater movement speed than those in Groups $D_{5}$ and $D_{10}$ is consistent with the findings of Rieber and our previous study. They have found that competing responses significantly affect the movement speed. In the light of these studies, the result for the movement speed in the present study may also be explained in terms of response competition in much the same way as for the result of the starting speed, as mentioned above. There is no difference between Groups $D_{5}$ and $D_{10}$ in the movement speed because competing responses do not affect movement speed as much as they affect starting speed as Spence has suggested (1956).

With regard to the effect of the incentive value, the present study fails to find significant relationship between this variable and the movement speed. This fact is not only inconsistent with findings of our previous and Rabinowitz's studies, in which $S \mathrm{~s}$ in the high incentive groups have shown a greater movement speed than those in the low incentive groups, but also inconsistent with the Spence's theory that the movement speed mainly reflects the effect of motivational variables (1956). A possible reason the incentive variable can not have entered into any significant relationship for the movement speed is that a string used as a low incentive object in the present study may be acting as a rather high incentive. During the experiment, some of the $S \mathrm{~s}$ in Groups $\mathrm{L}$ have shown such verbal behavior as expressing their greater interests in the string, for example, "What shall I play with this string after finishing my game?" or "Is it true that you have promised to give me this string?" Such a possibility has been suggested in a study reported by Sheikh (1968). He has used a method of the control in the incentive variable similar to that of the present study and has failed to obtain the effect of the incentive variable on the speed of the children's lever-pulling response. The discrepancies in the results between Rabinowitz and our previous study, and the present study may also be due to the above possibility, or due to differences in controls of incentive variables between these studies. In case of using a method of the control in the incentive-value variable similar to that of the present study, it is important that future studies pay attention to select appropriate incentive objects, especially low incentive object.

\section{REFERENCES}

LindQuist, E. F. 1953 Design and analysis of experiments. Boston, Mass.: Houghton Mifflin.

Rabinowitz, F. M. 1966 Conditioned stimulus duration and delay of reward as variables in a lever pulling situation. Fournal of Experimental Child Psychology, 3, 225-234.

Rieber, M. 1961 The effect of CS presence during delay of reward on the speed of an instrumental response. Fournal of Experimental Psychology, 61, 290-294.

ShetkH, A. A. 1968 Children's response speed as a function of delay of reward at different distances from the goal and incentive value. Psychonomic Science, 11, 201.

SHIRorWA, Y. 1975 Effects of delay of reinforcement and incentive value on children's lever pulling response. Fapanese Psychological Research, 17, 17-24.

Spence, K. W. 1956 Behavior theory and conditioning. New Haven: Yale University Press.

(Received July 25, 1975) 